\title{
Binding Time Analysis for Polymorphically Typed Higher Order Languages
}

\author{
Torben $\rightleftarrows$. Mogensen ${ }^{*}$ \\ DIKU \\ Computer Science Department \\ University of Copenhagen \\ Universitetsparken 1 \\ DK-2100 København $\varnothing$, DENMARK \\ email: torbenm@diku.dk
}

\begin{abstract}
Binding time analysis is used to distinguish the variables in a program that will have a known value at a time when only some of the parameters to the program are given. This is used in partial evaluation and has been found to be essential for efficient self-application and thus compiler generation by self-application of a partial evaluator with respect to an interpreter.

Until recently, works in binding time analysis have used first order untyped languages, but the last year have seen a few papers treating typed lambda-calculus and a paper that argues that projections (domain retracts) could be used to describe binding times in a first order typed functional language.

This paper extends the latter to higher order polymorphically typed functional languages and addresses some practical aspects of implementing the analysis.
\end{abstract}

Keywords: binding time analysis, compiler generation, functional languages, partial evaluation, projections, semantics.

\section{Introduction}

Binding time analysis determines when the variables of a program are bound to their values. A typical distinction is compile time versus run time. When using partial evaluation for compiler generation [Jones, Sestoft,S $\varnothing n d e r g a a r d$ 85,88], [Romanenko 88] explicit binding time annotations are essential, as argued in [Bondorf,Jones,Mogensen,Sestoft 88]. Nielson \& Nielson argue in [Nielson,Nielson 86] that binding time information is important when generating compilers from denotational semantics. Binding time analysis will be able to provide the necessary information.

Binding time analysis is essentially a dual problem to strictness analysis. Where strictness analysis finds how much of the parameters of a function is needed to produce a certain part of the result, binding time analysis finds how much of the result will be known, given which parts of the parameters are known. Experience shows that precision is more important in binding time analysis than in strictness analysis. An intuitive understanding of this can be found by considering the interdependence between functions in a program: given $f \circ g$, lets assume that a strictness analyzer fails to recognize that $f$ is strict, meaning that $g$ is in a lazy context. Even so, the strictness analyzer may find that $g$ is strict, since it need not consider the case where $g$ is not called (the code for $g$ will not be called in that case). But if a binding time analyzer fails to recognize that the result of $g$ is known, even if only part of the input is known, it will be assumed that $f$ is called with an unknown argument, and will thus (very likely) have an unknown result. In this way

\footnotetext{
*This work was mostly done at Imperial College of Science and Tectunology in London, partially supported by the Danish research
} academy 
an imprecise result will be propagated throughout the program being analyzed. Another reason for requiring better precision in binding time analysis is the purpose of the analysis: determining which computations should be done at compile time. Moving computations from compile time to run time can have disastrous effects on the efficiency of a program, whereas using lazy evaluation instead of strict evaluation has a more limited penalty.

Most previous work in binding time analysis [Jones,Sestoft,Søndergaard 85,88], [Jones 88], [Mogensen 88], [Romanenko 88] have used untyped first order languages. The notable exceptions are [Nielson,Nielson 88] and [Schmidt 88] that uses typed lambda calculus and [Launchbury 88] that uses a first order typed functional language. This paper is in one way best comparable to Schmidt's and Nielson \& Nielson's works as it uses a higher order language, but the method used is derived from Launchbury's use of projections (domain retracts) to describe binding times. Launchbury's paper describe the domain constructions and proves some properties about them, giving only hints as to how to actually perform the analysis. This paper examines this problem in details, and finds that there are some non-trivial problems involved, especially in connection with recursive data types and higher order types.

The goal of this paper is to provide an analysis with very precise results, aiming at a higher information content than the analysis of Nielson \& Nielson.

\section{Outline}

In section 2 we present the type system and the principle of using projections for describing binding times. In section 3 we construct finite projection domains for each type in the system and define the greatest lower bound of projections. Section 4 presents the binding time analysis algorithm by constructing abstract versions of the operators in a functional language and proving these correct. Section 5 shows two examples of the analysis, section 6 discuss some implementation issues and in section 7 we round off with some conclusions.

\section{Preliminaries}

Since the binding time analysis is intended to be used in connection with partial evaluation of a language similar to the modern strongly typed functional languages like Lazy ML or Miranda, we will in this paper use a simplified version of such languages, essentially typed curried combinators.

We use the Hindley/Milner type system as used in several functional languages (e.g. Lazy ML). This is a type system with sum, product, higher order types and polymorphism which we describe using an explicit fixed-point operator $(\mu)$ to construct recursive types, rather than by referencing to names in a global recursive type definition. We use this notation:

$$
\tau=\operatorname{void} \mid \text { int }|\ldots| \tau \times \tau\left|\operatorname{tag}_{1} \tau_{1}+\cdots+\operatorname{tag}_{n} \tau_{n}\right| \tau \rightarrow \tau\left|\mu \alpha_{i} \cdot \tau\right| \alpha_{i}
$$

where $\alpha_{i}$ are type variables and int is the type of integers. There can be other base types. The product is non-strict to allow lazy evaluation, and the sum is separated. void is an one-element type containing only $\perp$. Type variables bound by a $\mu$ are used for recursive types, and free type variables are used for polymorphism. The free variables are implicitly universally quantified. To restrict this to the Hindley/Milner type system (as known from e.g. ML), not all type formulas will be legal. Recursive types must be sum types (to lift the domain, it might be a "sum of one type"), and the injection tags used for sum types must be unique (i.e., no two types can use the same tag). In addition to this we require that there must be finite polymorphism, i.e., there can only be finitely many instantiations of polymorphic type variables. This is no great restriction, as extremely few programs use unbounded polymorphism. The Hindley/Milner type inferencer will only accept finitely polymorphic functions, if explicit user declarations of the types of functions are not used. 
A polymorphic list can with this notation be expressed as:

$$
\alpha \text { list }=\mu \beta: \text { nil void }+ \text { cons } \alpha \times \beta
$$

where $\alpha$, being a free type variable, is used for polymorphism.

John Launchbury [Launchbury 88] used projections to describe binding times. A projection $p_{a}$ is a retract, i.e., a mapping from a domain $A$ to itself such that:

$$
\begin{aligned}
& p_{a} \sqsubseteq I D_{A} \\
& p_{a} \circ p_{a}=p_{a}
\end{aligned}
$$

where $I D_{A}$ denotes the identity mapping on $A$. Projections can be used to describe information content: a projection $\left(I D_{A}\right)$ that maps all elements of $A$ to themselves, describes full information, whereas a projection $\left(A B S E N T_{A}\right)$ that maps all elements to $\perp$ will describe total lack of information. Thus it is natural to use projections to describe the amount of information available at partial evaluation time (comple time): whatever is left intact by a projection is considered static, whereas the parts mapped to $\perp$ are considered dynamic.

Binding time analysis consists of finding a projection py for the output of a function, given the function $f: A \rightarrow B$, and a projection $p_{a}$ for its input, so that

$$
p_{b} \circ f \circ p_{a}=p_{b} \circ f
$$

This means that whatever $p_{b}$ retains of the output of $f$ is not dependent of the part of input that $p_{a}$ discards. Note that we are interested in the $p_{b}$ that discards as little as possible of its value, i.e., the largest if we use the normal partial ordering of functions.

It might seem strange that we want to find the greatest solution, as it is normal in abstract interpretation to look for the least solution. However projections are not the same as sets of values, rather a projection describes information content. When using projections (with the normal ordering), it would seem natural in a forwards analysis to look for the strongest post-condition (i.e., the greatest solution) and in a backwards analysis to look for the weakest precondition (i.e., the least solution), as in strictness analysis using projections [Wadler,Hughes 87]. The choice between forwards and backwards analysis will depend on the problem. Strictness analysis "which parameters need to be known to find the result of the function" seems to be a kind of pre-condition, hereas the $p_{b}$ above represents a post-condition for $f$ with respect to the pre-condition represented by $p_{a}$.

Note that it will in general not be possible to find a maximal $p_{b}$. Consider the function:

$$
f x=\text { if } g x \text { then } x \text { else } x
$$

where we assume that the conditional is strict in the condition. If $g x$ never terminates then $f=\lambda x .1$, so $p_{b}=I D$ is safe by the equation above. If $g x$ sometimes terminates $p_{b}=p_{a}$ is the largest safe result. Thus, if $p_{a} \neq I D$ then finding the largest $p_{b}$ would involve solving the halting problem! We will in our analysis assume termination of programs, so the results of the analysis will be the same for strict and lazy languages.

The result of the binding time analysis can be used by a partial evaluator, either by annotating expressions with their binding time, as in [Jones, Sestoft, Sondergaard 86,88 ] or by transforming the program to separate the binding times, as in [Mogensen 89].

\section{Projections}

Given a type formula for a type $A$, we want to construct a finite domain of representations of projections $\mathcal{P}_{A}$ for $A$. $A n y$ $\mathcal{P}_{A}$ should contain representations of $I D_{A}$ and $A B S E N T_{A}$, where $A B S E N T_{A}=\lambda x \cdot \perp_{A} . I D$ and $A B S E N T$ will be 
used polymorphically to represent $I D_{A}$ and $A B S E N T_{A}$ for any $\mathrm{A}$. We will assume the existence of an implicit semantic function, mapping representations to projections. We allow several representations of the same projection, but we will be able to identify these. We will in the descriptions below allow ourselves to be a bit sloppy in distinguishing projections and their representations. When the difference is important, we will state which meaning is used. We will order the representations by comparing the projections they represent.

$$
\begin{aligned}
& \mathcal{P}_{\text {void }}=\{I D\}=\{A B S E N T\} \\
& \mathcal{P}_{\text {int }}=\{I D, A B S E N T\} \\
& \mathcal{P}_{A \times B}=\left\{\langle f, g\rangle \mid f \in \mathcal{P}_{A}, g \in \mathcal{P}_{B}\right\}
\end{aligned}
$$

where $\langle f, g\rangle$ represents $\lambda(a, b) .(f a, g b)$.

$$
\mathcal{P}_{\operatorname{tag}_{1} A_{1}+\cdots+\operatorname{tag} g_{n} A_{n}}=\{A B S E N T\} \cup\left\{\operatorname{tag}_{1}^{\prime} f_{1} \mp \cdots \mp \operatorname{tag}_{n}^{\prime} f_{n} \mid f_{i} \in \mathcal{P}_{A_{2}}\right\}
$$

Where we use $\operatorname{tag}_{1}^{\prime} f_{1} \mp \ldots \mp t a g_{n}^{\prime} f_{n}$ to represent

$$
\lambda x . c a s e x \text { of } \operatorname{tag}_{1} v: \operatorname{tag}_{1}\left(f_{1} v\right) ; \ldots \operatorname{tag}_{n} v: \operatorname{tag}_{n}\left(f_{n} v\right)
$$

where the case expression is strict in its sum-type argument (i.e., case $\perp$ of $\ldots=\perp$ ). Note that

$$
\begin{gathered}
I D_{\operatorname{tag} A_{1}+\cdots+\operatorname{tag}_{n} A_{n}}=\operatorname{tag}_{1}^{\prime} I D_{A_{1}} \mp \cdots \mp \operatorname{tag}_{n}^{\prime} I D_{A_{n}} \\
A B S E N T_{\operatorname{tag} 1} A_{1}+\cdots+\operatorname{tag}_{n} A_{n}
\end{gathered}
$$

For recursive types we have:

$$
\begin{array}{ll}
\mathcal{P}_{\mu \alpha_{1} A} & =\left\{f i x \lambda f_{i} \cdot p \mid p \in \mathcal{P}_{A}\right\} \\
\mathcal{P}_{\alpha_{z}} & =\left\{f_{i}\right\}
\end{array} \quad \text { if } \alpha_{i} \text { is bound by a } \mu
$$

Here we require consistency in the choice of identifiers. Free type variables have no fixed set of projections, rather they inherit the projection domain of any type that instantiates them ${ }^{1}$. The above definitions gives the following set of projections for the $\alpha$ list type:

$$
\mathcal{P}_{\alpha \text { list }}=\{f i x \lambda g . A B S E N T\} \cup\left\{f i x \lambda g . n i l^{\prime} I D \mp \operatorname{cons}^{\prime}\langle f, g\rangle \mid f \in \mathcal{P}_{\alpha}\right\}
$$

So the projection will either return $\perp$, or map a projection onto the elements of the list.

So far we have not defined projections on functional types. A projection on a function must return a smaller function: a function that always returns a smaller value. This can be done in several ways, it e.g.,. by composing it with a projection (in either end). It is however more useful (as we will see) to think of the function as a closure, and then replace parts of the closure by $\perp$. If we have the program in named combinator form, all free variables are converted to parameters, so a closure is just a function name and a partial list of parameters. A projection $\left(A B S E N T_{A \rightarrow B}\right)$ can replace the complete closure by $\perp$, or another projection can, depending on which function name the closure contains, apply projections to the parameters, giving a projection of the form:

$$
\begin{aligned}
& \text { גc. case } c \text { of } \\
& {\left[f v_{1} \ldots v_{n}\right]:\left[f\left(p_{1} v_{1}\right) \ldots\left(p_{n} v_{n}\right)\right]} \\
& {\left[g v_{1} \ldots v_{m}\right]:\left[g\left(q_{1} v_{1}\right) \ldots\left(q_{m} v_{m}\right)\right]}
\end{aligned}
$$

which can be represented by a set of abstract closures:

$$
\left\{\left[f p_{1} \ldots p_{n}\right], \ldots,\left[g q_{1} \ldots q_{m}\right]\right\}
$$

\footnotetext{
${ }^{1}$ This is only safe becanse we assume finite polymorphism. Otherwise we might generate infinitely many projections during binding time analysis.
} 
In this way the domain of projections for higher order types are not only dependent on the type, but also on the program. The set need not contain closures for all functions in the program, as it can be a default that any closure not represented will have the identity projection.

Whereas the projections for the first order values have a meaning independent of the program being analyzed, thus being extensional, the projection for functional values is strongly dependent on the actual program text, thus being very intensional. This makes the analysis less "clean", but it also greatly increases the quality of the result of the analysis. We believe that this increased precision is worth the price paid in loss of conceptual clarity.

\section{Greatest Lower Bound}

During a binding time analysis we will often want, given two or more projections, to find a projection that discards all that any of these discards and keeps all that all of these keep. This is the greatest lower bound $(\Pi)$ of the projections. The greatest lower bound of two projections $p_{1}$ and $p_{2}$ can be defined as:

$$
p_{1} \sqcap p_{2}=\lambda x \cdot\left(p_{1} x\right) \sqcap\left(p_{2} x\right)
$$

Note that in general the greatest lower bound of two projections need not be idempotent, and thus not a projection. In the domains of representations we will, however, be able to construct a representation of a lower bound that will represent a projection, and that (with the exception of the functional types) will represent the greatest lower bound in the domains of projections as well.

We will now construct the greatest lower bound for each $\mathcal{P}_{A}$.

For all types and all projections $p$ we will have:

$$
\begin{gathered}
I D \sqcap p=p \\
A B S E N T \sqcap p=A B S E N T
\end{gathered}
$$

This completely defines $\Pi$ for the types void and int, so we will continue with the remaining types, showing only the cases not defined by the above rules.

$$
\begin{gathered}
\mathcal{P}_{A \times B}: \\
\left\langle p_{a 1}, p_{b 1}\right\rangle \sqcap\left\langle p_{a 2}, p_{b 2}\right\rangle=\left\langle p_{a 1} \sqcap p_{a 2}, p_{b 1} \sqcap p_{b 2}\right\rangle \\
\mathcal{P}_{\operatorname{tag}_{1} A_{1}+\cdots+\operatorname{tag}_{n} A_{n}:} \\
\operatorname{tag}_{1} p_{a_{11}} \mp \ldots \mp \operatorname{tag}_{n} p_{a_{1 n}} \sqcap \operatorname{tag}_{1} p_{a_{21}} \mp \ldots \mp \operatorname{tag}_{n} p_{a_{2 n}}= \\
\operatorname{tag}_{1}\left(p_{a_{11}} \sqcap p_{a_{21}}\right) \bar{\mp} \ldots \mp \operatorname{tag}_{n}\left(p_{a_{1 n}} \Pi p_{a_{2 n}}\right)
\end{gathered}
$$

All these are trivial. For recursive types we have:

$$
\begin{aligned}
& \mathcal{P}_{\mu \alpha_{i}: A}: \\
& f i x \lambda f_{i} \cdot p_{a 1} \sqcap f i x \lambda g_{i} \cdot p_{a 2}=f i x \lambda h_{i} \cdot\left(p_{a 1}\left[f_{i} \backslash h_{i}\right] \sqcap p_{a 2}\left[g_{i} \backslash h_{i}\right]\right) \\
& \mathcal{P}_{\alpha_{2}}: \\
& h_{i} \sqcap h_{i}=h_{i}
\end{aligned}
$$

In the rule for a bound type variable we can assume that the same identifier is used, as a preceding use of the rule for recursive types will have performed alpha-conversion to ensure this. The rule for recursive types requires that $n$ is continuous in the domains of representations of projections. It is trivially monotonous, so only the limits of chains needs to be investigated. For the finite domains this is again trivial, and for the function space projections it can be proven by induction (omitted here). 
For projections on functions we have:

$$
\begin{aligned}
& \mathcal{P}_{A \rightarrow B}: \\
& p_{1} \cap p_{2}=\left\{c\left|\wedge p_{2}\right| d \in p_{1}\right\} \cup\left\{d \wedge p_{1} \mid d \in p_{2}\right\} \\
& \text { where } \\
& \text { otherwise } \\
& {\left[f p_{11} \ldots p_{1 n}\right] \wedge p=} \\
& {\left[f\left(p_{12} \sqcap p_{21}\right) \ldots\left(p_{n} \sqcap p_{2 n}\right)\right]} \\
& {\left[f p_{11} \ldots p_{1 n}\right]} \\
& \text { if there is a closure }\left[f p_{21} \ldots p_{2 n}\right] \in p
\end{aligned}
$$

It is easy to see that the constructed projection $p_{3}=p_{1} \sqcap p_{2}$ is in $\mathcal{P}_{A \rightarrow B}$ if the arguments $\left(p_{1}, p_{2}\right)$ are. To see that it is the greatest lower bound of these consider the result of applying $p_{i}$ to a closure $\left[g v_{1} \ldots v_{n}\right]$. If there is no abstract closure of the right structure in either of $p_{1}$ or $p_{2}$, there will not be in $p_{3}$ either, so all of them would map the closure to itself. If there are appropriate abstract closures $\left[g p_{11} \ldots p_{1 n}\right]$ and $\left[g p_{21} \ldots p_{2 n}\right]$ in $p_{1}$ and $p_{2}$ then there is an abstract closure $\left[g\left(p_{11} \Pi p_{21}\right) \ldots\left(p_{1 n} \Pi p_{2 n}\right)\right]$ in $p_{3}$. Hence what we need to show is that

$$
\begin{gathered}
{\left[g\left(p_{11} v_{1}\right) \ldots\left(p_{1 n} v_{n}\right)\right] \sqcap\left[g\left(p_{21} v_{1}\right) \ldots\left(p_{2 n} v_{n}\right)\right]=} \\
{\left[g\left(\left(p_{11} \sqcap p_{21}\right) v_{1}\right) \ldots\left(\left(p_{1 n} \Pi p_{2 n}\right) v_{n}\right)\right]}
\end{gathered}
$$

If the closure is seen as a data structure, this is certainly true. If it is seen as a function, that, is not necessarily the case. If $g$ is monotonic we have

$$
\begin{gathered}
{\left[g\left(p_{11} v_{1}\right) \ldots\left(p_{1 n} v_{n}\right)\right] \sqcap\left[g\left(p_{21} v_{1}\right) \ldots\left(p_{2 n} v_{n}\right)\right] \sqsupseteq} \\
{\left[g\left(\left(p_{11} \cap p_{21}\right) v_{1}\right) \ldots\left(\left(p_{1 n} \sqcap p_{2 n}\right) v_{n}\right)\right]}
\end{gathered}
$$

but the converse is not always true. Thus we have a lower bound, but not necessarily the greatest. It is however the greatest in the required form. Also, the functions $g$ for which it isn't the greatest lower bound would behave similar to parallel $O R$, and are thus not expressible in lambda calculus. So for the language presented below, the construction will indeed yield the greatest lower bound.

\section{Binding Time Analysis}

Binding time analysis consists as mentioned earlier of, given a definition of a function and a projection for its input, to find a projection for its output so that

$$
p_{b} \circ f \circ p_{a}=p_{b} \circ f
$$

where $p_{a}$ is the projection for the input to $f$ and $p_{b}$ is the projection for the output. As mentioned in section 2 , it will not be possible to find a maximal safe $p_{b}$, so we will just try to find a safe $p_{b}$ while taking care not to make it needlessly small. This will be done by constructing an abstract function $f^{\#}$ for each function $f$, such that $f^{\#} p_{a}=p_{b}$, where $p_{b}$ is the greatest projection in $\mathcal{P}_{B}$ that has the required property. The $f^{*}$ is constructed by replacing each operator op in $f$ 's definition by an abstract operator $o p^{\#}$. We must for each op prove that it has the correct relation to op. We will do this by first defining the syntax, then for each operator in the expression syntax define the abstract operator, and then prove that the properties hold.

$$
\begin{aligned}
<\text { program }>::= & f_{1}<\text { variable }_{11}>\ldots<\text { variable }_{1 n_{1}}>=<\exp _{1}> \\
& \ldots \\
& f_{m}<\text { variable }_{m 1}>\ldots<\text { variable }_{m n_{m}}>=\left\langle\exp _{m}>\right.
\end{aligned}
$$




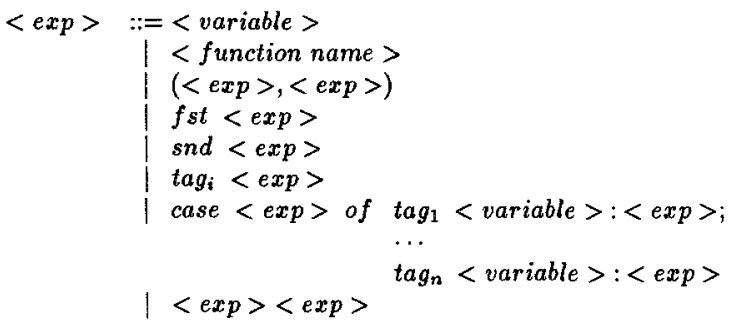

Thus we can define $f_{i}^{\#}$ as:

$$
f^{\#} p_{x_{1}} \ldots p_{x_{n_{i}}}=e_{i}^{\#}
$$

where $f_{i}$ is defined by

$$
f_{i} x_{1} \ldots x_{n_{3}}=e_{i}
$$

and $e_{i}^{\#}$ is $e_{i}$ where all operators have been replaced by their abstract (hashed) counterpart, all variables $x_{j}$ have been replaced by projection variables $p_{x_{j}}$ and all named functions $f_{k}$ by a constant singleton set of abstract closures $\left\{\left[f_{k}\right]\right\}$. The operators can be given polymorphic types:

$$
\begin{aligned}
& (-,-): A \times B \rightarrow A \times B \\
& f \text { st }: A \times B \rightarrow A \\
& \text { snd }: A \times B \rightarrow B \\
& \operatorname{tag}_{i}: A_{i} \rightarrow\left(\operatorname{tag}_{1} A_{1}+\cdots+\operatorname{tag}_{n} A_{n}\right) \\
& \text { case }:\left(A_{1} \rightarrow B\right) \times \cdots \times\left(A_{n} \rightarrow B\right) \rightarrow\left(\operatorname{tag}_{1} A_{1}+\cdots+\operatorname{tag}_{n} A_{n}\right) \rightarrow B \\
& \text { apply }:(A \rightarrow B) \times A \rightarrow B
\end{aligned}
$$

where we look at the branches of the case operator as functions of the "pattern" variables. Note that the $\operatorname{tag}_{i}$ and case operators are not really polymorphic in the sum type arguments. This is because we required unique injection tags, so we could say we have a family of case functions. The branches of a case expression are seen as functions from the summand types to the result type. These functions are seen as a part of the case operator. The apply operator is invisible in the actual syntax, where $\langle\exp \rangle\langle\exp \rangle$ is application of a function to an argument. In addition to these, we will also have constants and operations on the base types $\left(1,2,^{\prime} a^{\prime},+,-, \ldots\right)$. We can now give types for the abstract operators:

$$
\begin{array}{ll}
(-,-)^{\# \#} & : \mathcal{P}_{A} \times \mathcal{P}_{B} \rightarrow \mathcal{P}_{A \times B} \\
f_{s t}^{\#} & : \mathcal{P}_{A \times B} \rightarrow \mathcal{P}_{A} \\
\text { snd }^{\#} & : \mathcal{P}_{A \times B} \rightarrow \mathcal{P}_{B} \\
\text { tagi }_{i}^{\#} & : \mathcal{P}_{A_{i}} \rightarrow \mathcal{P}_{\text {tag }_{1}} A_{1}+\cdots+\operatorname{tag}_{n} A_{n} \\
\text { case\# } & :\left(\mathcal{P}_{A_{1}} \rightarrow \mathcal{P}_{B}\right) \times \cdots \times\left(\mathcal{P}_{A_{n}} \rightarrow \mathcal{P}_{B}\right) \rightarrow \mathcal{P}_{\text {tag }_{1} A_{1}+\cdots+\operatorname{tag}_{n} A_{n}} \rightarrow \mathcal{P}_{B} \\
\text { apply } & : \mathcal{P}_{A \rightarrow B} \times \mathcal{P}_{A} \rightarrow \mathcal{P}_{B}
\end{array}
$$

Note that the parameters of case could expect. This is because the branches in case\# will be abstract expressions (built from abstract operators), and thus projection transformers rather than projections of value transformers. We will now give definitions of the abstract operators. The abstract version of a base type constant is the identity projection and the abstract version of a strict base type operator (like,$+ *$ etc.) returns $A B S E N T$ unless all parameters are $I D$, in which case it returns $I D$

$$
\begin{aligned}
& (-,-)^{\#}=\lambda\left(p_{a}, p_{b}\right) \cdot\left\langle p_{a}, p_{b}\right\rangle \\
& f s t^{\#}=\lambda p_{a b} \cdot p_{a} \text { where } p_{a b}=\left\langle p_{a}, p_{b}\right\rangle \\
& s n d^{\#}=\lambda p_{a b} \cdot p_{b} \text { where } p_{a b}=\left\langle p_{a}, p_{b}\right\rangle \\
& t a g_{i}^{\#}=\lambda p_{a} \cdot t a g_{1}^{\prime} I D \mp \ldots \mp t a g_{i}^{\prime} p_{a} \mp \ldots \mp t a g_{n}^{\prime} I D \\
& \text { case }^{\#}=\lambda\left(F_{a_{1} b}^{\#}, \ldots, F_{a_{n b}}^{\#}\right) \cdot \lambda p_{a} \text {. } \\
& \text { case } p_{a} \text { of } \\
& \text { ABSENT :ABSENT } \\
& \operatorname{tag}_{1}^{\prime} f_{1} \mp \ldots \mp \operatorname{tag}_{n}^{\prime} f_{n}:\left(F_{a_{1} b}^{\#} f_{1}\right) \Pi \cdots \Pi\left(F_{a_{n} b}^{\#} f_{n}\right)
\end{aligned}
$$


In the abstract expressions variables (e.g. $x$ ) have been replaced by projection identifiers (e.g. $p_{x}$ ). Function names (e.g. $f$ ) are replaced by projection constants (e.g. $\{[\mathrm{f}]\})$. The rules have assumed the absence of $f i x$ in the projections.

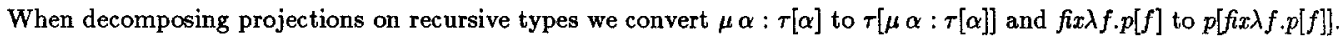
As $f i x$ is used only on sum type projections, it is only case \# that has to worry about this. Similarly it is only $t a g_{i}^{\#}$ that have to worry about introducing $f i x$. We will use the fact that the tags are unique to find the type they inject into, specifically if it is a recursive type. In case it is we will extract the projection corresponding to the type variable by the method below, and create a new recursive projection by taking the greatest lower bound of this and the projection obtained by replacing the extracted parts by a projection identifier (f) using the replace function below, and adding a fix $f$ around the resulting projection.

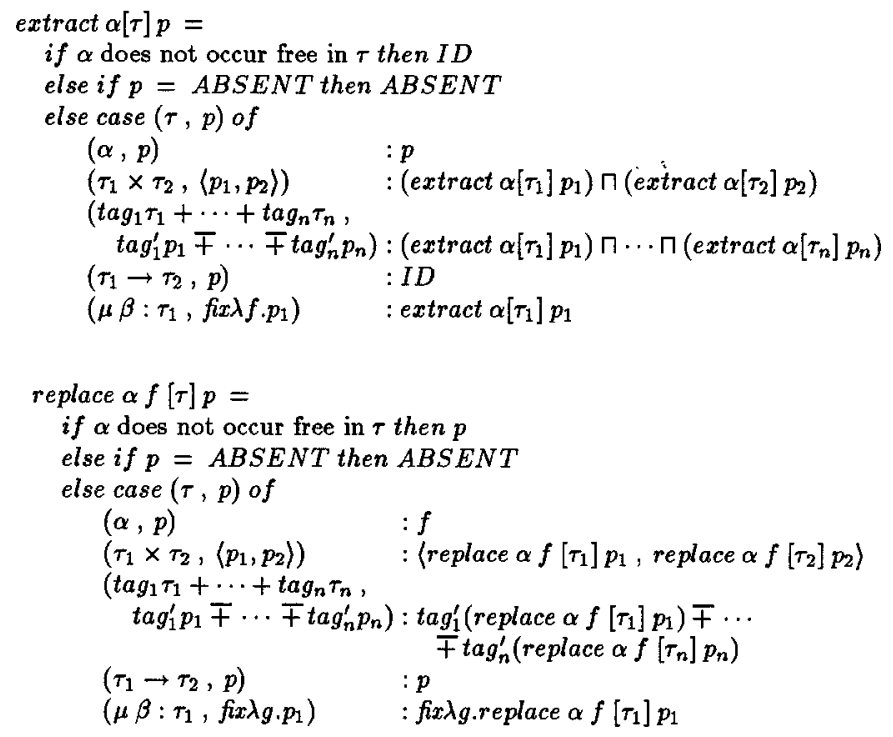

apply $y^{\#}$ must have the property:

$$
a p p l y^{\#}\left(p_{a b}, p_{a}\right) \circ\left(p_{a b} f\right) \circ p_{a}=a p p l y^{\#}\left(p_{a b}, p_{a}\right) \circ f
$$

for any function $f: A \rightarrow B$ and any projection $p_{a} \in \mathcal{P}_{A}$. If this should hold for any imaginable $f$, it is fairly easy to see that $a p p l y^{\#}\left(p_{a b}, p_{b}\right)=A B S E N T$ unless both $p_{a b}$ and $p_{a}$ are the identity projections (in which case it is $I D$ ). But by relaxing the condition to hold for only the functions that actually occur in a given program, we can obtain better results. For the previously mentioned projection structure we can define:

$$
\begin{aligned}
& a p p l y^{\#}\left(p_{a b}, p_{a}\right)=\begin{array}{l}
A B S E N T \\
\prod_{c \in p_{a b}}^{A} a p p_{1}^{\#} c p_{a} \text { otherwise }
\end{array} \\
& \text { where } \\
& \qquad a p p_{1}^{\#}\left[f p_{1} \ldots p_{n}\right] p_{a}=\begin{array}{ll}
f^{\#} p_{1} \ldots p_{n} p_{a} & \text { if } f \text { has } n+1 \text { parameters } \\
\left\{\left[f p_{1} \ldots p_{n} p_{a}\right]\right\} & \text { otherwise }
\end{array}
\end{aligned}
$$

Essentially we add parameters to all the closures involved, producing new closures. When the final element in a closure is given we use the abstract function $f^{\#}$ on the argument projections giving a result projection. Since it is possible that some of the abstract closures will be given their final argument and others not, we produce a result projection for each closure (which can be just by adding a parameter to the closure) and take the greatest lower bound of these.

This definition gives us

$$
a p p l y^{\#}\left(\{[f]\}, p_{a}\right)=f^{\#} p_{a}
$$


for first-order functions $f$, as one would expect. $f^{\#}$ must be defined to handle polymorphism. This is done by letting it be polymorphic over projection domains. As mentioned above the type system ensures that there will only be finitely many instantiations of any given polymorphic type, so this will not cause non-termination.

\section{Correctness}

To prove the definitions of $o p^{\#}$ correct, we must first prove that for an operator op: $A \rightarrow B$ that

$$
p_{b}=o p^{\#} p_{a} \Rightarrow p_{b} \circ \circ \circ \circ p_{a}=p_{b} \circ o p
$$

for all $p_{a}$ (the safety criterion). The abstract versions of base type constants and operators are trivially correct, so we will continue straight away with the remaining cases:

$$
\begin{aligned}
& o p=(-,-): p_{b}=(-,)^{\#}\left(p_{a 1}, p_{a 2}\right)=\left\langle p_{a 1}, p_{a 2}\right\rangle \\
& p_{b} \circ(-,-) \circ\left\langle p_{a 1}, p_{a 2}\right\rangle \\
& =\left\langle p_{a 1}, p_{a 2}\right\rangle \circ\left\langle p_{a 1}, p_{a 2}\right\rangle \\
& =\left\langle p_{a 1} \circ p_{a 1}, p_{a 2} \circ p_{a 2}\right\rangle \\
& =\left\langle p_{a 1}, p_{a 2}\right\rangle \\
& =\left\langle p_{a 1}, p_{a 2}\right\rangle \circ(-,-) \\
& o p=f s t: p_{b}=f s t^{\#} p_{a}=p_{a 1} \text { where } p_{a}=\left\langle p_{a 1}, p_{a 2}\right\rangle \\
& p_{b} \circ f s t \circ p_{a} \\
& =p_{a 1} \circ f s t \circ\left\langle p_{a 1}, p_{a 2}\right\rangle \\
& =p_{a 1} \circ f s t \circ\left(\lambda(x, y) \cdot\left(p_{a 1} x, p_{a 2} y\right)\right) \\
& =p_{a 1} \circ\left(\lambda(x, y) \cdot p_{a 1} x\right) \\
& =p_{a 1} \circ p_{a 1} \circ(\lambda(x, y) \cdot x) \\
& =p_{a 1} \circ f s t \\
& \text { 口 } \\
& o p=s n d: p_{b}=s n d^{\#} p_{a}=p_{a 2} \text { where } p_{a}=\left\langle p_{a 1}, p_{a 2}\right\rangle \\
& p_{b} \circ \text { snd } \circ p_{a} \\
& =p_{a 2} \circ \text { snd } \circ\left\langle p_{a 1}, p_{a 2}\right\rangle \\
& =p_{a 2} \circ \text { snd } \circ\left(\lambda(x, y) \cdot\left(p_{a 1} x, p_{a 2} y\right)\right) \\
& =p_{a 2} \circ\left(\lambda(x, y) \cdot p_{a 2} y\right) \\
& =p_{a 2} \circ p_{a 2} \circ(\lambda(x, y) \cdot y) \\
& =p_{a 2} \circ \text { snd } \\
& \square \\
& o p=\operatorname{tag}_{i}: p_{b}=\operatorname{tag}_{i}^{\#} p_{a}=\operatorname{tag}_{1}^{\prime} I D \mp \ldots \mp \operatorname{tag} g_{i}^{\prime} p_{a} \mp \ldots \mp \operatorname{tag} g_{n}^{\prime} I D
\end{aligned}
$$

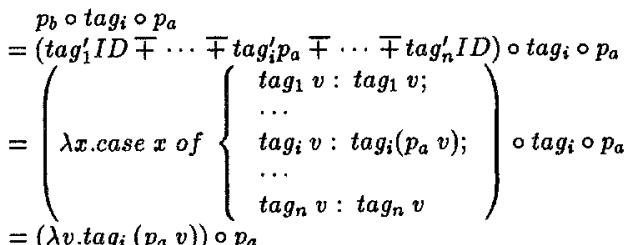

$$
\begin{aligned}
& =\left(\lambda v \cdot \operatorname{tag}_{i}\left(p_{a} v\right)\right) \circ p_{a} \\
& =\operatorname{tag}_{i} \circ p_{a} \circ p_{a} \\
& =\operatorname{tag}_{i} \circ p_{a} \\
& =p_{b} \circ \operatorname{tag}_{i}
\end{aligned}
$$

For the case operator we will look at case $\left(f_{1}, \ldots, f_{n}\right)$ and the corresponding case\# $\left(f_{1}^{\#}, \ldots, g_{1}^{\#}\right)$. We will consider the functions $f_{i}$ as part of the case operator and the abstract functions $f_{i}^{\#}$ as part of the case $\#$ operator. We will 
assume by induction:

$$
\left(f_{i}^{\#} p_{a}\right) \circ f_{i} \circ p_{a}=\left(f_{i}^{\#} p_{a}\right) \circ f_{i}
$$

for all $f_{i}$ and all $p_{a}$. We will make use of a lemma:

$$
f \sqsubseteq g \Rightarrow f \circ g=f \quad \text { for all projections } f \text { and } g
$$

proof:

$$
\begin{aligned}
& f \subseteq g \\
\Rightarrow & f \circ f \subseteq f \circ g \\
\Rightarrow & f \sqsubseteq f \circ g \\
& g \subseteq I D \\
\Rightarrow & f \circ g \sqsubseteq f \circ I D \\
\Rightarrow & f \circ g \subseteq f
\end{aligned}
$$

We will start with the case where $p_{a}=A B S E N T$ :

$$
\begin{aligned}
o p= & \operatorname{case}\left(f_{1}, \ldots, f_{n}\right): \\
p_{b}= & \operatorname{case}{ }^{\#}\left(f_{1}^{\#}, \ldots, f_{n}^{\#}\right) \text { ABSENT }=A B S E N T \\
& p_{b} \circ \operatorname{case}\left(f_{1}, \ldots, f_{n}\right) \circ p_{a} \\
= & A B S E N T \circ \operatorname{case}\left(f_{1}, \ldots, f_{n}\right) \circ A B S E N T \\
= & A B S E N T \\
= & A B S E N T \circ \operatorname{case}\left(f_{1}, \ldots, f_{n}\right) \\
& \square
\end{aligned}
$$

and then the case where $p_{a}=t a g_{1}^{\prime} p_{a_{1}} \mp \ldots \mp \operatorname{tag} g_{n}^{\prime} p_{a_{n}}$ :

$$
\begin{aligned}
& o p=\operatorname{case}\left(f_{1}, \ldots, f_{n}\right): \\
& p_{b}=\operatorname{case}^{\#}\left(f_{1}^{\#}, \ldots, f_{n}^{\#}\right)\left(\operatorname{tag}_{1} p_{a_{1}} \mp \ldots \mp \operatorname{tag}_{n} p_{a_{n}}\right) \\
& =\left(f_{1}^{\#} p_{a_{1}}\right) \Pi \cdots \Pi\left(f_{n}^{\#} p_{a_{n}}\right) \\
& p_{b} \circ \operatorname{case}\left(f_{1}, \ldots, f_{n}\right) \circ\left(\operatorname{tag}_{1} p_{a_{1}} \mp \ldots \mp \operatorname{tag} p_{n} p_{a_{n}}\right) \\
& =p_{b} \circ\left(\lambda x \text {.case } x \text { of } \operatorname{tag}_{1} v: f_{1} v ; \ldots \operatorname{tag}_{n} v: f_{n} v\right) \\
& \text { o }\left(\lambda x \text { case } x \text { of } \operatorname{tag}_{1} v: \operatorname{tag}_{1}\left(p_{a_{1}} v\right)_{;} \ldots \operatorname{tag}_{n} v: \operatorname{tag}_{n}\left(p_{a_{n}} v\right)\right) \\
& =p_{b} \circ\left(\lambda x \text {.case } 2 \text { of } \operatorname{tag}_{1} v: f_{1}\left(p_{a_{1}} v\right) ; \ldots \operatorname{tag}_{n} v: f_{n}\left(p_{a_{n}} v\right)\right) \\
& =\left(\lambda x \text {.case } x \text { of } \operatorname{tag}_{1} v: p_{b} \circ f_{1} \circ p_{a_{1}} v ; \ldots \operatorname{tag}_{n} v: p_{b} \circ f_{n} \circ p_{a_{n}} v\right) \\
& =\left(\lambda x . \text { case } x \text { of } \operatorname{tag}_{1} v: p_{b} \circ\left(f_{1}^{\#} p_{a_{1}}\right) \circ f_{1} \circ p_{a_{1}} v\right. \text {; } \\
& \text {... } \\
& \left.\operatorname{tag}_{n} v: p_{b} \circ\left(f_{n}^{\#} p_{a_{n}}\right) \circ f_{n} \circ p_{a_{n}} v\right) \\
& =\left(\lambda x . \text { case } x \text { of } \operatorname{tag}_{1} v: p_{b} \circ\left(f_{1}^{\#} p_{a_{1}}\right) \circ f_{1} v\right. \text {; } \\
& \left.\operatorname{tag}_{n} v: p_{v} \circ\left(f_{n}^{\#} p_{a_{n}}\right) \circ f_{n} v\right) \\
& =\left(\lambda x \text {.case } x \circ f \operatorname{tag}_{1} v: p_{b} \circ f_{1} v ; \ldots \operatorname{tag} g_{n} v: p_{b} \circ f_{n} v\right) \\
& =p_{b} \circ\left(\lambda x \text {.case } x \text { of } \operatorname{tag}_{1} v: f_{1} v ; \ldots \operatorname{tag}_{n} v: f_{n} v\right) \\
& =p_{b} \circ \text { case }\left(f_{1}, \ldots, f_{n}\right) \\
& \square
\end{aligned}
$$

Proving safety for apply $y^{\#}$ is proving the property:

$$
a p p l y^{\#}\left(p_{a b}, p_{a}\right) \circ\left(p_{a b} f\right) \circ p_{a}=a p p l y^{\#}\left(p_{a b}, p_{a}\right) \circ f
$$

for any functional value $f: A \rightarrow B$ that can occur while executing the program. If the function projection $p_{a b}$ is $A B S E N T$ then $a p p l y^{\#}\left(p_{a b}, p_{a}\right)$ is $A B S E N T$ as well, making the property trivially true. Otherwise that $f$ is a closure $\left[g v_{1} \ldots v_{n}\right]$, and $p_{a b}$ is a set of abstract closures. Then $p_{a b} f$ is $\left[g\left(p_{1} v_{1}\right) \ldots\left(p_{n} v_{n}\right]\right.$ where $\left[g p_{1} \ldots p_{n}\right]$ is the abstract closure for $g$ in $p_{a b}$, or $[g I D \ldots I D]$ if there is no such abstract closure. apply $\#\left(p_{a b}, p_{a}\right)$ will be less than $a p p_{1}^{\#}\left[g p_{1} \ldots p_{n}\right] p_{a}$, so by the lemma given above, all we have to prove is that

$$
\begin{aligned}
& \left(a p p_{1}^{\#}\left[g p_{1} \ldots p_{n}\right] p_{a}\right) \circ\left[g\left(p_{1} v_{1}\right) \ldots\left(p_{n} v_{n}\right)\right] \circ p_{a}= \\
& \left(a p p_{1}^{\#}\left[g p_{1} \ldots p_{n}\right] p_{a}\right) \circ\left[g v_{1} \ldots v_{n}\right]
\end{aligned}
$$


We will consider the two cases: $g$ has $n+1$ parameters, and $g$ has more than $n+1$ parameters. In the first case

$$
a p p_{1}^{\#}\left[g p_{1} \ldots p_{n}\right] p_{a}=g^{\#} p_{1} \ldots p_{n} p_{a}
$$

and

$$
\left[g\left(p_{1} v_{1}\right) \ldots\left(p_{n} v_{n}\right)\right] \circ p_{a}=\lambda v_{a} \cdot g\left(p_{1} v_{1}\right) \ldots\left(p_{n} v_{n}\right)\left(p_{a} v_{a}\right)
$$

By induction we can assume that

$$
\left(g^{\#} p_{1} \ldots p_{n} p_{a}\right)\left(g\left(p_{1} v_{1}\right) \ldots\left(p_{n} v_{n}\right)\left(p_{a} v_{a}\right)\right)=\left(g^{\#} p_{1} \ldots p_{n} p_{a}\right)\left(g v_{1} \ldots v_{n} v_{a}\right)
$$

which is what we needed to prove the property. In the case of $g$ having more than $n+1$ parameters

$$
a p p_{1}^{\#}\left[g p_{1} \ldots p_{n}\right] p_{a}=\left\{\left[g p_{1} \ldots p_{n} p_{a}\right]\right\}
$$

and

$$
\left[g\left(p_{1} v_{1}\right) \ldots\left(p_{n} v_{n}\right)\right] \circ p_{a}=\lambda v_{a} \cdot\left[g\left(p_{1} v_{1}\right) \ldots\left(p_{n} v_{n}\right)\left(p_{a} v_{a}\right)\right]
$$

and since

$$
\begin{aligned}
& \left\{\left[g p_{1} \ldots p_{n} p_{a}\right]\right\}\left[g\left(p_{1} v_{1}\right) \ldots\left(p_{n} v_{n}\right)\left(p_{a} v_{a}\right)\right] \\
= & {\left[g\left(p_{1}\left(p_{1} v_{1}\right)\right) \ldots\left(p_{n}\left(p_{n} v_{n}\right)\right)\left(p_{a}\left(p_{a} v_{a}\right)\right)\right] } \\
= & {\left[g\left(p_{1} v_{1}\right) \ldots\left(p_{n} v_{n}\right)\left(p_{a} v_{a}\right)\right] } \\
= & \left\{\left[g p_{1} \ldots p_{n} p_{a}\right]\right\}\left[g v_{1} \ldots v_{n} v_{a}\right]
\end{aligned}
$$

we have what we want.

\section{Recursive function space projections}

A function space projection may contain abstract closures that have parameters that again are abstract closures etc., so an analysis might run infinitely by building larger and larger nested structures. To solve this we propose to approximate infinite nested structures by recursive definitions. This will be done by recognizing similarities between different levels in the closures and construct a recursive definition by identifying the levels. This might lead to a less precise (smaller) projection, but will not make a safe projection unsafe, so the correctness proofs will still be valid.

When a recursive projection is used it is unfolded one step (which will not change the value). After application the result is made recursive again if necessary.

For recursive types the type structure determines when recursion needs to be introduced in the projection. For functional types this is not so easy. However, abstract closures can only be infinitely nested if a function's arguments can contain a closure of that same function. This can to some extent be decided by the type structure, in the sense that the type of a function must allow a parameter that contain a function of the same type. There are several ways of tying the recursion. One is to make a recursive definition every time the type would make it possible, another is to do it when a closure actually occur nested inside one of the same structure. The tighter we tie the recursion, the less information we obtain, but the analysis is likely to be faster, as we have made the set of possible values smaller. We have chosen a compromise: if in a set of closures, one of the closures contain a set of closures that overlap the top-level set, then make the set recursive. The recursion is made by replacing the inner set of closures by an identifier, adding the extracted closures to the top level and making the projection recursive in the inserted variable.

\section{Fixed-point iteration}

Binding time analysis will be done by a fixed-point iteration using minimal function graphs [Jones, Mycroft 86]. In this strategy we use a minimal function graph (MFG) which contain mappings of abstract functions to their result for specific argument sets (those that are actually needed). The initial MFG will contain only one mapping: the goal 
(abstract) function with its parameters, binding the result to the top value (ID). Iteration proceeds by re-evaluating all mappings in the MFG. When a function call is needed, its value from the MFG is used. If there is no mapping for a call, one is added that maps the call to $I D$. If re-evaluation gives a result different from the one in the MFG, the mapping is changed to the greatest lower bound of the previous and the new value. As all changes are monotonic and there are only finitely many possible argument value combinations the iteration will terminate when a fixed-point is reached. This will then be a consistent solution, and it will be the largest possible as values start with the top element (ID) and are only made lower when necessary.

\section{Examples}

An example using polymorphism is shown below. First we define functions:

$$
\begin{aligned}
& \alpha \text { list }=\mu \beta: \text { nil void }+ \text { cons } \alpha \times \beta \\
& f:(\alpha \text { list }) \rightarrow((\alpha \times \text { int }) \times \text { int list }) \\
& f x=m(m x) \\
& m:(\alpha \text { list }) \rightarrow(\alpha \times \text { int list }) \\
& m x=\text { case } x \text { of nil } v: \text { nil } v ; \\
& \text { cons } v: \text { cons }((f s t v, 42), m(\text { snd } v))
\end{aligned}
$$

Then the abstract functions:

$$
\begin{aligned}
& f^{\#:}: \mathcal{P}_{(\alpha \text { list })} \rightarrow \mathcal{P}_{((\alpha \times \text { int }) \times \text { int list })} \\
& f^{\#} p_{x}=m^{\#}\left(m^{\#} p_{x}\right) \\
& m^{\#}: \mathcal{P}_{(\alpha l i s t)} \rightarrow \mathcal{P}_{(\alpha \times \text { int list })} \\
& m^{\#} p_{x}=\text { case \# } p_{x} \text { of nil } v: n i l^{\#} v ; \\
& \quad \text { cons } v: \text { cons }
\end{aligned}
$$

Note that we have called $m^{\#}$ directly instead of using apply\# which we can do, as $m$ is first order.

To find $f^{\#}$ fix $\lambda g . A B S E N T$ we construct an initial minimal function graph (MFG):

$$
\left[\left(f^{\#} f i x \lambda g \cdot A B S E N T \mapsto I D\right)\right]
$$

Initially assuming the result is $I D$. During the fixed-point iteration we add function calls to the MFG, initially mapping them to $I D$. When we evaluate them we change their value to the new value. This yields the following sequence in the iteration:

$$
\begin{aligned}
& {\left[\left(f^{\#} f i x \lambda g \cdot A B S E N T \mapsto I D\right)\right]} \\
& {\left[\left(f^{\#} f i x \lambda g . A B S E N T \mapsto I D\right),\right.} \\
& \left.\left(m^{\#} f i x \lambda g . A B S E N T \mapsto I D\right)\right] \\
& {\left[\left(f^{\#} \text { fix } \lambda g . A B S E N T \mapsto I D\right),\right.} \\
& (m \text { \# } f x \lambda g . A B S E N T \mapsto A B S E N T)] \\
& {\left[\left(f^{\#} f i x \lambda g . A B S E N T \mapsto A B S E N T\right),\right.} \\
& \left.\left(m^{\#} f i x \lambda g . A B S E N T \mapsto A B S E N T\right)\right] \\
& {\left[\left(f^{\#} f i x \lambda g . A B S E N T \mapsto A B S E N T\right),\right.}
\end{aligned}
$$

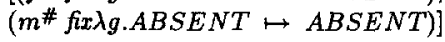

As expected, changing the elements of a completely unknown list results in a completely unknown list. if we start with a list of unknown elements we get the following final result: 


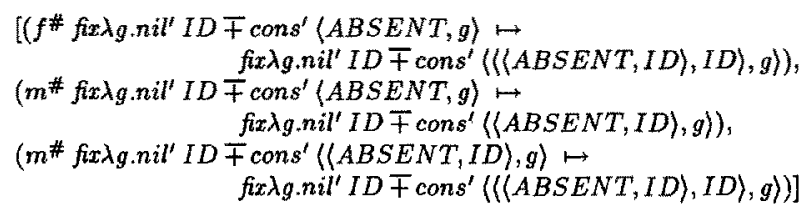

where we have omitted the intermediate steps for reasons of space. Note that the two mappings for $m^{\#}$ are of different instances of the polymorphic type. There need not always be a mapping for every instance, as all mappings where the projection for the polymorphic part is $I D$ or $A B S E N T$ will be shared by all types.

Now we will show an example with higher order functions. In the example closures can be built to an arbitrary depth, which means that recursive function projections will be needed.

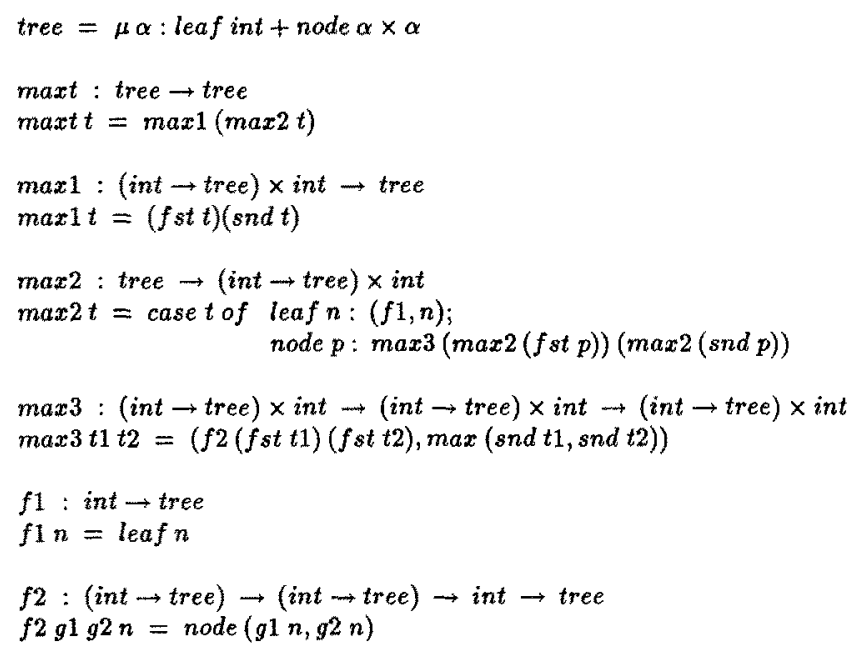

The function maxt takes as argument a binary tree with integer leaves and returns a tree with the same structure but with all the leaves replaced by the maximum value of the leaves of the input tree. This is done by constructing a function that given an integer will build the tree with that integer and then supply this with the maximum value that was found in the same passage of the input tree as the function. Now follows the abstract functions (this time without type declarations):

$$
\begin{aligned}
& \max t^{\#} t=\max 1^{\#}\left(\max 2^{\#} t\right) \\
& \max 11^{\#} t=\operatorname{apply} y^{\#}\left(f s t^{\#} t\right)\left(s n d^{\#} t\right) \\
& \max 2^{\#} t=\operatorname{case}^{\#} t \text { of } \\
& \text { leaf } n:\langle\{[f 1]\}, n\rangle \\
& \text { node } p: \max 3^{\#}\left(\max 2^{\#}\left(f s t^{\#} p\right)\right)\left(\max 2^{\#}\left(s n d^{\#} p\right)\right) \\
& \max 3^{\#} t 1 t 2=\left\langle a p p l y^{\#}\{[f 2]\}\left(f s t^{\#} t 1\right)\left(f s t^{\#} t 2\right),\left(s n d^{\#} t 1\right) \cap\left(s n d^{\#} t 2\right)\right) \\
& f 1^{\#} n=\text { leaf }{ }^{\#} n \\
& f 2^{\#} g 1 g 2 n=\text { node }\left\langle a p p l y * g 1 n, a p p l y y^{\#} g 2 n\right\rangle
\end{aligned}
$$

In the iteration below we start by giving maxt a projection that keeps the structure of the tree but discards the leaves. We would thus expect a result projection of the same type. This projection has the form:

$$
\text { fix } \lambda p_{\alpha} \text { leaf } f^{\prime} A B S E N T \mp \text { node' }\left\langle p_{\alpha}, p_{\alpha}\right\rangle
$$


and will be referred to as $p p$ in the example. We show only the final result, and have removed unreachable configurations.

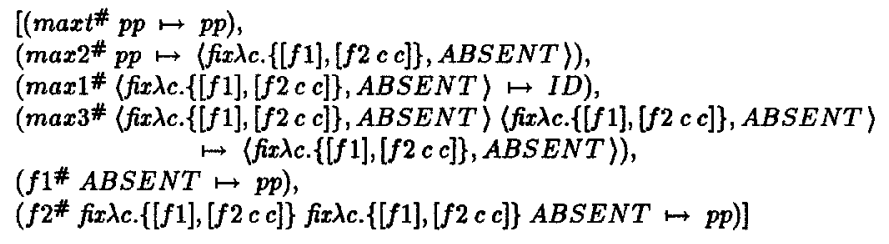

This gave the expected result for the result of maxt. The interesting part is however the result of max2 which shows a recursive function projection. If we couldn't make recursive closures the iteration would yield larger and larger nonrecursive nested approximations.

\section{Implementation Issues}

In the rules for greatest lower bound and in other places we have either assumed that there are no name conflicts in the variables used to make recursive types and projections or else explicitly alpha-converted the names. In an implementation this can be be made simpler by using DeBruijn indexing of these variables. This also makes comparison of projections easier.

In some cases where higher order functions are used to build continuations the minimal function graph will contain separate mappings for all possible continuation structures (down to the depth where recursion is tied). As well as making the analysis slow, this also makes the result less useful, as we are really only interested in a single mapping using all possible continuations. A way of achieving this is to combine mappings in the MFG that have identical arguments except for the functional arguments, where the sets of closures are overlapping. The overlap requirement makes the chance of combining mappings that are really used in different contexts small. Note that the condition for combining mappings is the same as the condition for making a function projection recursive.

In the examples shown, the value of a mapping is always taken from the previous MFG, even if a new value has already been computed in the new MFG. Using the new value instead will make the iteration shorter. If this strategy is used, evaluating the latest additions to the MFG first will improve iteration speed further.

\section{Conclusion}

We have presented an algorithm for binding time analysis of a higher order functional language with a polymorphic type system. It extends the ideas of [Launchbury 88] to higher order functions and polymorphic types, and compares well to [Nielson,Nielson 88] by handling values that are partially static, containing both compile time and run time information in a single variable, which the present version of Nielson \& Nielson cannot do (though I believe their basic framework could be extended to do so). By restricting the projections domains to contain only $I D$ and $A B S E N T$, a result similar to that of Nielson \& Nielson can be obtained. The analysis is not as conceptually clean as Nielson \& Nielson's, but we believe the increased precision is worth the cost.

\section{Acknowledgements}

I would like to thank Chris Hankin for supervising my work at Imperial College and also many other people there for helpful discussions. I also had benefit from discussions with John Launchbury, John Hughes and several other people from the University of Glasgow. Finally thanks to Neil Jones, other people at DIKU and Flemming Nielson for helpful criticism and comments. 


\section{References}

[Bondorf, Jones, Mogensen, Sestoft 88]

A.Bondorf, N.D.Jones, T.A.Mogensen, P.Sestoft, Self-Application as a Tool for the Generation of Program Generators submitted for publication

[Jones 88]

N.D.Jones, Automatic Program Specialization: a Re-examination from Basic Principles in the Proceedings of the Workshop on Partial Evaluation and Mixed Computation, Denmark, October 1987, eds. D.Bjørner, A.P.Ershov and N.D.Jones, North-Holland 1988

[Jones, Mycroft 86]

N.D.Jones, A.Mycroft, Data Flow Analysis of Applicative Programs Using Minimal Function Graphs in the Proceedings of the Workshop on Programs as Data Objects, Copenhagen, October 1985, Springer Verlag LNCS 217, 1986

[Jones,Sestoft,Søndergaard 85]

N.D.Jones, P.Sestoft, H.Søndergaard, An Experiment in Partial Evaluation: the Generation of a Compiler Generator in Rewriting Techniques and Applications (ed. J.-P. Jouannaud), Dijon, France 1985, Springer Verlag LNCS 202, 1985

[Jones,Sestoft,Søndergaard 88]

N.D.Jones, P.Sestoft, H.S $\phi$ ndergaard, Mix: a Self-Applicable Partial Evaluator for Experiments in Compiler Generation in LISP and Symbolic Computation $13 / 4,1988$

[Launchbury 88]

J.Launchbury, Projections for Specialisation in the Proceedings of the Workshop on Partial Evaluation and Mixed Computation, Denmark, October 1987, eds. D.Bjørner, A.P.Ershov and N.D.Jones, North-Holland 1988

[Mogensen 88]

T.Æ.Mogensen, Partially Static Structures in a Self-Applicable Partial Evaluator in the Proceedings of the Workshop on Partial Evaluation and Mixed Computation, Denmark, October 1987, eds. D.Bjorner, A.P.Ershov and N.D.Jones, North-Holland 1988

[Mogensen 89]

T.Æ.Mogensen, Separating Binding Times in Language Specifications submitted for publishing

[Nielson, Nielson 86]

H.R. Nielson, F.Nielson, Semantics Directed Compiling for Functional Languages in the Proceedings of the ACM Conference on LISP and Functional Programming 1986

[Nielson, Nielson 88]

H.R.Nielson, F.Nielson, Automatic Binding Time Analysis for a Typed $\lambda$-Calculus in Science of Computer Programming 10, North-Holland 1988

[Romanenko 88]

S.A.Romanenko, A Compiler Generator Produced by a Self-Applicable Specialiser can have a Surprisingly Natural and Understandable Structure in the Proceedings of the Workshop on Partial Evaluation and Mixed Computation, Denmark, October 1987, eds. D.Bjørner, A.P.Ershov and N.D.Jones, North-Holland 1988

[Schmidt 88]

D.A.Schmidt, Static Properties of Partial Reduction in the Proceedings of the Workshop on Partial Evaluation and Mixed Computation, Denmark, October 1987, eds. D.Bjørner, A.P.Ershov and N.D.Jones, North-Holland 1988

[Wadler, Hughes 87]

P.Wadler, R.M.J.Hughes Projections for Strictness Analysis in the Proceedings of 1987 Functional Programming Languages and Computer Architecture Conference, Springer Verlag LNCS 274, September 1987 